\section{Biodegradable collagen matrix implant vs mitomycin-C as an adjuvant in trabeculectomy: a 24-month, randomized clinical trial}

Department of Experimental Biomedicine and Clinical Neuroscience,

Ophthalmology Section, University of Palermo (Italy) Palermo, Italy

Correspondence: S Cillino, Department of Experimental Biomedicine and Clinical Neuroscience,

Ophthalmology Section, University of Palermo (Italy) Via Liborio Giuffrè 13, 90127 Palermo, Italy Tel: + 39916553929 Fax: + 39916261438 . E-mail: cillino@unipa.it

Received: 10 February 2011 Accepted in revised form: 19 July 2011

Published online:

16 September 2011

\begin{abstract}
Aim To verify the safety and efficacy of Ologen (OLO) implant as adjuvant compared with low-dosage mitomycin-C (MMC) in trabeculectomy.

Methods This was a prospective randomized clinical trial with a 24-month follow-up. Forty glaucoma patients (40 eyes) were assigned to trabeculectomy with MMC or OLO. Primary outcome includes target IOP at $\leq 21, \leq 17$, and $\leq 15 \mathrm{~mm} \mathrm{Hg}$; complete (target IOP without medications), and qualified success (target IOP regardless of medications). Secondary outcomes include bleb evaluation, according to Moorfields Bleb Grading System (MBGS); spectral domain optical coherence tomography (SD-OCT) examination; number of glaucoma medications; and frequency of postoperative adjunctive procedures and complications.

Results The mean preoperative IOP was 26.5 $( \pm 5.2)$ in MMC and $27.3( \pm 6.0)$ in OLO eyes, without statistical significance. One-day postoperatively, the IOP dropped to $5.2( \pm 3.5)$ and $9.2( \pm 5.5) \mathrm{mm} \mathrm{Hg}$, respectively $(P=0.009)$. The IOP reduction was significant at end point in all groups $(P=0.01)$, with a mean IOP of 16.0 $( \pm 2.9)$ and $16.5( \pm 2.1) \mathrm{mm} \mathrm{Hg}$ in MMC and OLO, respectively. The rates and Kaplan-Meier curves did not differ for both complete and qualified success at any target IOP. The bleb height in OLO group was higher than MMC one $(P<0.05)$. SD-OCT analysis of successful/ unsuccessful bleb in patients with or without complete success at IOP $\leq 17 \mathrm{~mm} \mathrm{Hg}$ indicated a sensitivity of $83 \%$ and $73 \%$ and a specificity of
\end{abstract}

S Cillino, F Di Pace, G Cillino and A Casuccio

$75 \%$ and $67 \%$, respectively, for MMC and OLO groups. No adverse reaction to OLO was noted. Conclusions Our results suggest that OLO implant could be a new, safe, and effective alternative to MMC, with similar long-term success rate.

Eye (2011) 25, 1598-1606; doi:10.1038/eye.2011.219; published online 16 September 2011

Keywords: trabeculectomy; mitomycin-C; Ologen; adjuvants in trabeculectomy

Introduction

Many adjunctive modifications, such as antimetabolites, amniotic membrane transplantation, and expanded polytetrafluoroethylene (Gore-Tex) implants, have been tried in order to enhance and maintain the hypotensive effect of trabeculectomy. ${ }^{1-10}$

In various studies, adjunctive mitomycin- $\mathrm{C}$ (MMC) has been demonstrated to increase the success rate of trabeculectomy when compared with trabeculectomy alone or with postoperative 5-fluorouracil injections. ${ }^{1,2,11-13}$

Unfortunately, antimetabolite-augmented glaucoma surgery can be associated with a higher frequency of prolonged wound leaks, hypotony with choroidal effusions and maculopathy, thin avascular blebs, and/or bleb leaks with late infection. ${ }^{9,14,15}$

Recently, a new biodegradable porous collagen-glycosaminoglycan (GAG) copolymer 
matrix implant has been proposed for glaucoma surgery on the basis of animal experiments. ${ }^{16-18}$ Ologen (OLO) collagen matrix (Aeon Astron Europe BV, Leiden, The Netherlands) is a biodegradable collagen-GAG implant, available in various shapes and dimensions, that may maintain the hypotensive effect in glaucoma surgery and enhance the healing in other selected ophthalmic surgeries (eg, pterygium or strabismus). According to the manufacturer, when the OLO is directly placed over the scleral flap, its porous structure should force conjunctival fibroblasts and myofibroblasts to grow into the pores and secrete connective tissue in the form of a loose matrix, reducing scar formation and wound contraction. After the implantation, the device should completely degrade within 90-180 days.

A prospective, interventional case series with OLO implantation for open-angle glaucoma demonstrated that lower IOP correlates with bleb height, thin bleb wall, large subconjunctival fluid spaces, and low bleb tissue reflectivity. ${ }^{19}$ A prospective, randomized, medium-term pilot study failed to show any IOP-lowering advantage of the OLO and indicated a tendency to a higher incidence of complications with the collagen implant. ${ }^{20}$ Recent data from a randomized study of trabeculectomy using MMC vs an OLO implant showed a lower complete success rate but a lower bleb-associated complication rate in OLO group. ${ }^{21}$

The purpose of this randomized, prospective clinical trial was to compare the outcomes of trabeculectomy using either the adjunctive OLO implant or intraoperative, low-dosage MMC. The parameters measured included IOP, bleb morphology, and frequency of complications.

\section{Patients and methods}

This study was a prospective randomized phase II clinical trial undertaken in the Department of Ophthalmology of Palermo University, Italy, between January and December 2008. The protocol of this study was approved by the Ethics Committee of University of Palermo (Italy). Patients were thoroughly informed about the procedures and written informed consent was obtained in accord with tenets of Declaration of Helsinki.

Forty consecutive Caucasian glaucoma patients were randomly assigned to undergo a trabeculectomy with MMC (MMC group) or a trabeculectomy with OLO implant (OLO group). Randomization was determined just before surgery by sealed-envelope technique based on their surgical chart number. The sequence of random allocation was generated by pulling 40 standard sized pieces of paper out of a hat by the trial statistician (AC). Twenty pieces of paper were marked with letter A, and 20 with letter B. Each piece of paper was sequentially placed into 40 sealed, opaque envelopes by the trial statistician. The sealed envelopes were numbered 1 to 40 and given to the surgeon (SC). Patients were numbered randomly from 1 to 40 based on a surgical chart number related to the baseline testing session and intervention period. The clinical data collecting and measurement of outcome variables were performed by skilled personnel (ophthalmologists and optometrists) masked to randomization and who had not been directly involved in patient surgery.

Inclusion criteria were age 18 or older, diagnosis of POAG or pseudoexfoliative glaucoma (PEXG), and inadequate IOP control (IOP $>21 \mathrm{~mm} \mathrm{Hg}$ ) or progressive visual field deterioration on maximum-tolerated medical therapy. Exclusion criteria were normal-tension glaucoma, use of systemic or ocular medications that might affect vision, acute or chronic disease that could confound the outcomes of the study (eg, immunodeficiency, connective tissue disease, and diabetes), clinically significant cataract where combined surgery was indicated, and history of ocular trauma or prior ocular surgery.

The preoperative data collected were age; gender; medical history, including the presence of any ocular pathology; number of antiglaucomatous drugs used; applanation tonometry under maximum-tolerated topical therapy; biomicroscopy; and computerized Humphrey visual field testing (mean deviation and pattern standard deviation).

IOP was the primary outcome measure and three different IOP target levels were considered: $\leq 21, \leq 17$, and $\leq 15 \mathrm{~mm} \mathrm{Hg}$. Complete success was defined as a target end point IOP without antiglaucomatous medications, while qualified success was defined as a target end point IOP regardless of medications.

Secondary outcome measures included bleb evaluation, according to Moorfields Bleb Grading System (MBGS), which describes area, height, and vascularity of bleb; number of glaucoma medications; and frequency of postoperative adjunctive procedures and complications.

\section{Surgical techniques}

Patients underwent surgery on the second day of hospitalization. All operations were carried out under local peribulbar anesthesia by one experienced surgeon (SC). The technique included grasping the superior rectus muscle with a $4-0$ silk traction suture and creating a superior fornix-based conjunctival/tenons flap with a 9-mm limbal conjunctival incision using Westcott scissors. A rectangular $3.0 \times 3.5 \mathrm{~mm}^{2}$-wide, $300-\mu$ thick scleral flap was dissected at the 12-o'clock position using a bevel-up crescent knife (Alcon, Milan, Italy). The scleral 
flap $3.0 \mathrm{~mm}$ side incisions were not completed up to limbus. This should encourage greater posterior aqueous flow and a more diffuse bleb, according to the 'Moorfields Safer Surgery System' .22,23

When MMC was the randomized adjunctive therapy (Kyowa S.r.l., Milan, Italy), a Weck-cell sponge was cut into two to three pieces, $\sim 4 \mathrm{~mm} \times 2 \mathrm{~mm} \times 0.5 \mathrm{~mm}$, soaked with MMC at a concentration of $0.2 \mathrm{mg} / \mathrm{ml}$ and placed under the dissected conjunctiva surrounding the scleral flap ${ }^{22,23}$ and on the scleral bed. ${ }^{24}$ The sponges were left in position for $2 \mathrm{~min}$ in order to maintain contact with the Tenon's capsule side of conjunctiva. Thereafter, the eye was irrigated with $15 \mathrm{ml}$ of balanced salt solution. An ophthalmic viscoelastic (IAL-F, Bausch e Lomb IOM SpA, Milan, Italy) was injected to increase the iris-cornea depth and anterior chamber was entered at the base of scleral flap with a 3.2 precalibrated knife (Alcon Italia SpA). Two semicircular excisions $1.5 \mathrm{~mm}$ in diameter were created with a Crozafon-De Laage punch along the same radial line, in order to obtain an excision of corneoscleral tissue including the trabecular meshwork. A peripheral iridectomy was then performed, followed by reinjection of viscoelastic into the anterior chamber. The scleral flap was closed with two 10-0 nylon sutures, one at each corner, applying minimal tension in MMC cases and with one loose stitch in OLO cases. For the OLO cases, a cylindrical $2.0 \pm 0.3 \mathrm{~mm}$ in height $\times 6.0 \pm 0.5 \mathrm{~mm}$ in diameter implant (model number 830601, Aeon Astron Europe BV) was then centered on the top of scleral flap and under the conjunctiva. The conjunctival flap was secured to the limbus with two 10-0 nylon single-stitch tensioning sutures at the extremities of the limbal incision plus a tight 10-0 nylon running suture with buried knots. The filtration was assessed by injecting balanced salt solution into the paracentesis. Postoperatively, all eyes were treated with topical tobramycin $0.3 \%$ five times daily until day 14 , and with topical dexamethasone drops $0.1 \%$ five times daily for 7 days, three times daily for 6 weeks and twice a day for a final 1 week. If corkscrew bleb vessels were present, more frequent topical steroid administration was allowed, according to the intensified postoperative care' (IPC) protocol. ${ }^{25}$ Instillation of $1 \%$ atropine drops was added during the first few days, and continued up to 1 week in cases with hypotony. If needed, adjunctive procedures such as the Carlo Traverso maneuver ${ }^{26}$ (ie, applying a pressure with a cotton swab just temporal to the temporal radial groove of the sclerostomy, to disrupt the incision during the time of healing to try to prevent the sclerostomy from closing), laser suture lysis or bleb needling (without antimetabolites) were employed. If postoperative IOP measurements were $>21 \mathrm{~mm} \mathrm{Hg}$ after topical steroid withdrawal, IOP-lowering medication was added.
Postoperative visits were scheduled at $24 \pm 4 \mathrm{~h}, 7 \pm 1$ days, 2 weeks, and 1, 2, 3, 6, 12, 18, and 24 months. IOP (average of two separate readings), biomicroscopic findings, number of antiglaucomatous medications, and postoperative complications were assessed at each visit. Signs of inflammation, such as cells and flare, were graded from 0 to 4 , as were any bleb complications, such as cystic or avascular blebs.

At each follow-up visit, bleb photographs were recorded and graded according to the $\mathrm{MBGS}^{27}$ by a single observer (GC). Spectral domain optical coherence tomography (SD-OCT; Topcon 3DOCT-1000, Topcon Corporation, Tokyo, Japan) was performed at the end point for bleb evaluation by one ophthalmologist (FDP) masked to clinical data.

SD-OCT bleb analysis standardization was performed as follows. The blebs were classified as successful when the bleb wall was thickened (subjectively assessed relative to conjunctival-episcleral appearance) and discrete hyporeflective spaces or microcysts were visualized in the bleb wall. The presence or absence of bleb wall thickening and of the abovesaid intrableb wall structures, which identified a bleb as successful or failed, was assessed with respect to a $\leq 17 \mathrm{~mm} \mathrm{Hg}$ target IOP level. ${ }^{28,29}$ Thereafter, the sensitivity and specificity rates were calculated.

\section{Statistical analysis}

The sample size of 40 patients (20 eyes in each group) was chosen to achieve a power of $90 \%$ for detecting a $3 \mathrm{~mm} \mathrm{Hg}$ difference in IOP between treatment procedures, assuming a standard deviation of $3 \mathrm{~mm} \mathrm{Hg}$ and a two-sided $\alpha$ error of 5\%.

The independent Student's t-test and the MannWhitney $U$ statistic test were used for parametric and non-parametric analysis, respectively. Discrete variables were analyzed using the $\chi^{2}$ and Fisher's exact test, as needed. The paired-samples Student's $t$-test and paired Wilcoxon signed-rank test were used for intragroup parametric and non-parametric analysis, respectively. All statistical tests were two-tailed and were applied at the $5 \%$ significance level. Success was evaluated on the basis of Kaplan-Meier cumulative probability (log-rank test).

Before the study onset, we established an internal quality control system to assess intraobserver reproducibility and consistency by using three consecutive independent interpretations of the same SD-OCT scan, and by the use of the unweighted Cohen kappa $(\kappa)$ test. $^{30}$

Data were analyzed with Epi Info software, version 3.2.2 (Centers for Disease Control and Prevention, Atlanta, GA, USA) and SPSS software (version 14.0; SPSS Inc., Chicago, IL, USA). 


\section{Results}

All patients completed the 24-month follow-up period. Patients in the two treatment groups did not significantly differ in age, gender, type of glaucoma, mean preoperative values of visual field parameters, and mean number or duration of preoperative topical antiglaucomatous medication and preoperative IOP (Table 1).

The mean preoperative IOP $( \pm \mathrm{SD})$ was $26.5( \pm 5.2)$ in MMC eyes and $27.3( \pm 6.0)$ in OLO eyes, without significant intergroup difference. One-day postoperatively, the IOP dropped to $5.2( \pm 3.5)$ and 9.2 $( \pm 5.5) \mathrm{mm} \mathrm{Hg}$, respectively $(P=0.009)$. No intergroup difference was present at any scheduled postoperative observation time. The postoperative IOP reduction was still significant at the end point in all groups $(P=0.01)$, with a mean IOP of $16.0( \pm 2.9)$ and $16.5( \pm 2.1) \mathrm{mm} \mathrm{Hg}$ in MMC and OLO eyes, respectively. The percentage IOP reduction from baseline was 39.6 and 39.5, respectively (Table 2; Figure 1).

The success rates in the study groups are reported in Table 3. At $\leq 21 \mathrm{~mm} \mathrm{Hg}$ target IOP, complete success was achieved in 14 eyes (70\%) and 15 eyes (75\%) in MMC and OLO groups, respectively, without significant intergroup difference. At the same target IOP, a qualified success was achieved in 17 (85\%) and 18 (90\%) eyes, respectively, again without significant intergroup difference. A $\leq 17 \mathrm{~mm} \mathrm{Hg}$ target IOP complete success was obtained in $12(60 \%)$ and $11(55 \%)$ eyes in MMC and OLO groups, respectively, while a qualified success was reached by 15 eyes $(75 \%)$ in both groups. Finally, at $\leq 15 \mathrm{~mm} \mathrm{Hg}$ target IOP, 8 eyes $(40 \%)$ and 10 eyes (50\%) in MMC and OLO groups reached a complete success, while $12(60 \%)$ and $14(70 \%)$ eyes reached a qualified success, respectively, again without significant difference.

The Kaplan-Meier cumulative survival curves relating either the $\leq 21$, $\leq 17$, or $\leq 15 \mathrm{~mm} \mathrm{Hg}$ target IOP did not show significant intergroup differences for complete (log-rank $P=0.595,0.999$, and 0.349 , respectively) or qualified success rates (log-rank $P=0.131,0.794$, and 0.059, respectively) (Figure 2).

In order to allow for bleb stabilization, we performed MBGS scores comparison between the groups at 3 months, 6 months, 1 and 2 years. The central area, maximal area, and height score values (mean \pm SD) did not differ on an intragroup basis over time, maintaining a stable value till the end point in both surgical groups. The end point mean score values relating central area, maximal area, and height in MMC vs OLO group were $2.8 \pm 1.0$ vs $2.7 \pm 1.0,3.0 \pm 1.2$ vs $2.9 \pm 1.0$, and $1.3 \pm 0.7$ vs $1.6 \pm 0.8$, respectively. The same values, except for height, did not differ between the two groups at any time. In fact, the mean bleb height score was higher in OLO group at the third month $(2.0 \pm 0.8$ vs $1.3 \pm 0.7 ; P=0.009$; Mann-Whitney $U$ statistic test), maintaining a higher yet not significant value till the end point. The mean vascularity scores did not differ on an intragroup and intergroup basis over time. The end point central, peripheral, and non-bleb vascularity mean score values in MMC vs OLO group were $2.0 \pm 1.0$ vs $2.1 \pm 1.0,2.0 \pm 1.2$ vs $2.0 \pm 1.0$, and $2.0 \pm 1.0$ vs $2.0 \pm 1.0$, respectively. No cases with subconjunctival blood were recorded.

Table 2 Postoperative IOP $(\mathrm{mm} \mathrm{Hg})$ in the surgical groups from the 3rd month till end point

\begin{tabular}{lccc}
\hline & MMC group & OLO group & $\mathrm{P}^{\mathrm{a}}$ \\
\hline 3rd month & $14.7(3.9 ; 12.9-16.4)$ & $15.0(3.8 ; 13.3-16.7)$ & 0.806 \\
& $44.5 \%$ & $45.1 \%$ & \\
6th month & $14.7(4.3 ; 12.7-16.6)$ & $14.1(3.1 ; 12.6-15.4)$ & 0.615 \\
& $44.5 \%$ & $48.4 \%$ & \\
12th month & $15.0(3.0 ; 13.6-16.4)$ & $15.2(2.8 ; 13.8-16.4)$ & 0.828 \\
& $43.4 \%$ & $44.3 \%$ & \\
24th month & $16.0(2.9 ; 14.6-17.4)$ & $16.5(2.1 ; 15.5-17.4)$ & 0.536 \\
& $39.6 \%$ & $39.5 \%$ & \\
\hline
\end{tabular}

Abbreviations: MMC, mitomycin-C; OLO, Ologen.

andependent Student's $t$-test.

Mean ( \pm SD; $95 \%$ CI); \% change in IOP from baseline.

Table 1 Preoperative characteristics of patients

\begin{tabular}{|c|c|c|c|}
\hline & $M M C$ group & OLO group & $\mathrm{P}$ \\
\hline Gender $(\mathrm{M} / \mathrm{F}), N^{\circ}$ & $11 / 9$ & $12 / 8$ & $1.0^{\mathrm{a}}$ \\
\hline Age, years (mean $\pm S D)$ & $63.2(7.2)$ & $65.8(6.4)$ & $0.234^{\mathrm{b}}$ \\
\hline Right/left eyes, $N^{\circ}$ & $7 / 13$ & $11 / 9$ & $0.340^{\mathrm{a}}$ \\
\hline Type of glaucoma (POAG/PEXG), $N^{\circ}$ & $12 / 8$ & $13 / 7$ & $1.0^{\mathrm{a}}$ \\
\hline Preoperative IOP, $\mathrm{mm} \mathrm{Hg}($ mean \pm SD) & $26.5(5.2)$ & $27.3(6.0)$ & $0.654^{\mathrm{b}}$ \\
\hline Mean deviation, $\mathrm{dB}($ mean $\pm \mathrm{SD})$ & $-7.80(4.57)$ & $-7.41(5.35)$ & $0.805^{\mathrm{b}}$ \\
\hline Pattern standard deviation, $\mathrm{dB}$ (mean $\pm \mathrm{SD})$ & $7.20(4.10)$ & $7.0(4.23)$ & $0.880^{\mathrm{b}}$ \\
\hline Preoperative medications, $N^{\circ}($ mean $\pm \mathrm{SD})$ & $2.5(0.3)$ & $2.6(0.2)$ & $0.222^{\mathrm{b}}$ \\
\hline Duration of preoperative antiglaucoma therapy, years (mean $\pm S D$ ) & $5.7(1.8)$ & $6.3(1.4)$ & $0.246^{\mathrm{b}}$ \\
\hline
\end{tabular}

Abbreviations: MMC, mitomycin-C; OLO, Ologen; POAG, primary open angle glaucoma; PEXG, pseudoexfoliation glaucoma.

${ }^{\mathrm{a}} \chi^{2}$-test or Fisher's exact test, as needed.

'Independent Student's $t$-test. 


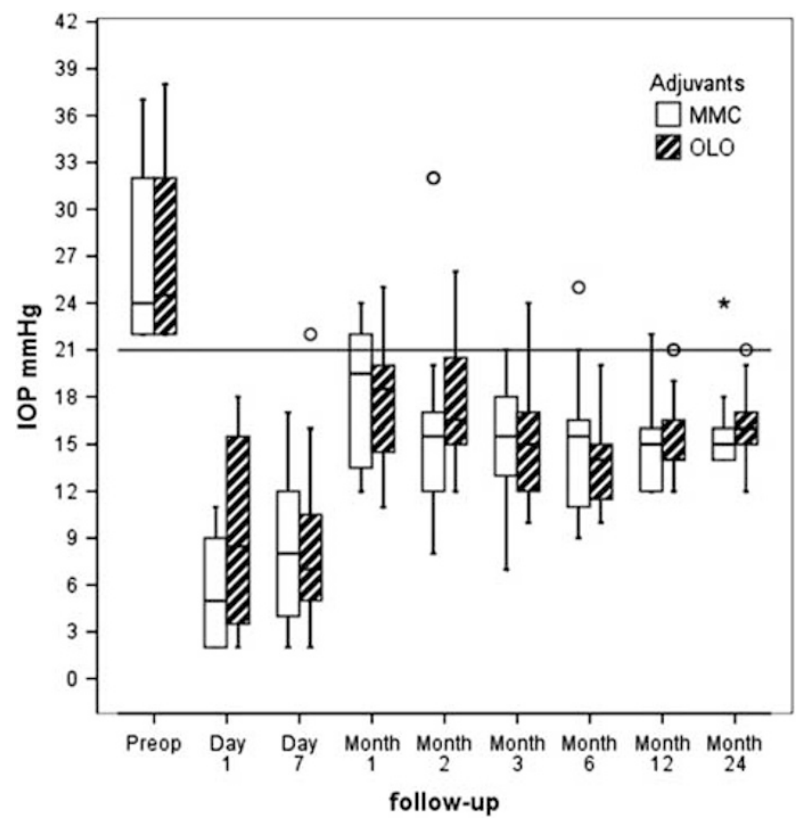

Figure 1 Box-plot representation of IOP values over 24 months of follow-up: median values (dark lines), error standard (T-bars), and outliers (circles).

Table 3 Success rates (\%) at the 24-month follow-up study end point in the surgical groups at three target IOP levels

\begin{tabular}{lccc}
\hline & MMC group & OLO group & $\mathrm{P}^{\mathrm{a}}$ \\
\hline$\leq 21 \mathrm{~mm} \mathrm{Hg}$ & & & \\
$\quad$ Complete success & $14(70 \%)$ & $15(75 \%)$ & 1.0 \\
Qualified success & $17(85 \%)$ & $18(90 \%)$ & 1.0 \\
& & & \\
$\leq 17 \mathrm{~mm} \mathrm{Hg}$ & $12(60 \%)$ & $11(55 \%)$ & 1.0 \\
Complete success & $15(75 \%)$ & $15(75 \%)$ & 1.0 \\
Qualified success & & & \\
& & $10(50 \%)$ & 0.751 \\
$\leq 15 \mathrm{~mm} H g$ & $8(40 \%)$ & $14(70 \%)$ & 0.741 \\
Complete success & $12(60 \%)$ & & \\
Qualified success & & & \\
\hline
\end{tabular}

Abbreviations: MMC, mitomycin-C; OLO, Ologen.

a'Fisher's exact test.

There was high intraobserver reproducibility for SD-OCT analysis ( $\kappa=0.7403,95 \%$ CI: 0.70-0.86). Table 4 shows the SD-OCT frequencies of thickened bleb wall and visualized discrete hyporeflective spaces (or microcysts), which identified a bleb as successful (Figure 3, top left and right), with respect to a $\leq 17 \mathrm{~mm} \mathrm{Hg}$ target IOP level at 2-year follow-up. A successful bleb was exhibited in 10 of 12 eyes with complete success based on IOP in the MMC group (Figure 3, top left), and in 8 of 11 in the OLO group (Figure 3, top right). These values represent a sensitivity of $83 \%$ and $73 \%$, respectively. Conversely, when considering the eyes without complete success, an

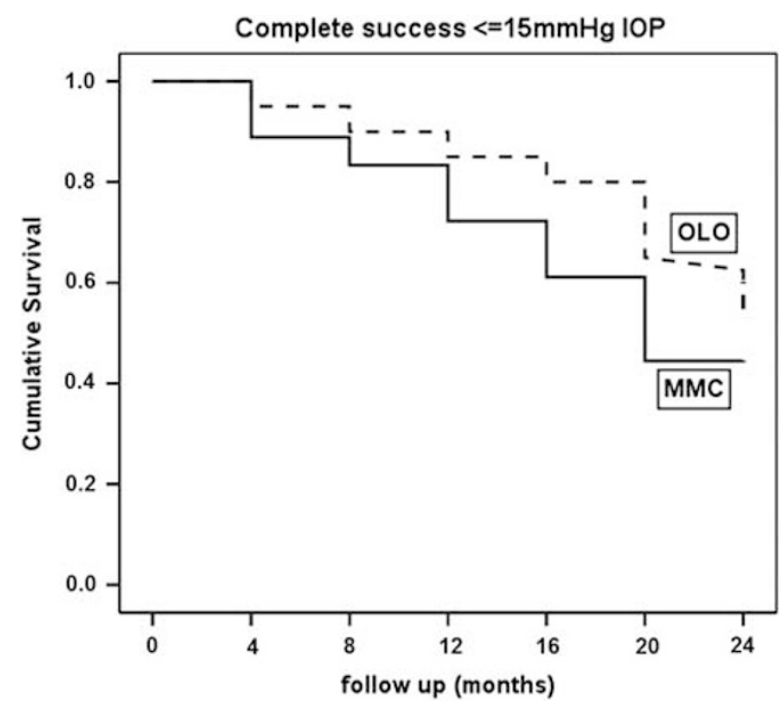

Figure 2 Kaplan-Meier cumulative probability curve of complete success (without medications) at $\leq 15 \mathrm{~mm} \mathrm{Hg}$ target IOP in MMC (solid line) vs OLO group (dotted line) (log-rank test $P=0.349)$.

Table 4 Bleb success rates (\%) at $\leq 17 \mathrm{~mm} \mathrm{Hg}$ target IOP in the surgical groups according to the SD-OCT analysis

\begin{tabular}{|c|c|c|c|}
\hline & $\begin{array}{l}\text { MMC } \\
\text { group }\end{array}$ & $\begin{array}{l}\text { OLO } \\
\text { group }\end{array}$ & $\mathrm{P}$ \\
\hline $\begin{array}{l}\text { Successful bleb/eyes with } \\
\text { complete success }\end{array}$ & $10 / 12$ & $8 / 11$ & $0.640^{\mathrm{a}}$ \\
\hline $\begin{array}{l}\text { Failed bleb/eyes without } \\
\text { complete success }\end{array}$ & $6 / 8$ & $6 / 9$ & $1.0^{\mathrm{a}}$ \\
\hline SD-OCT sensitivity & $83 \%$ & $73 \%$ & $0.871^{\mathrm{b}}$ \\
\hline SD-OCT specificity & $75 \%$ & $67 \%$ & $0.767^{\mathrm{b}}$ \\
\hline
\end{tabular}

Abbreviations: MMC, mitomycin-C; OLO, Ologen.

${ }^{a}$ Fisher's exact test.

${ }^{b} \chi^{2}$-test for the comparison of two proportions (from independent samples), expressed as a percentage.

SD-OCT-failed bleb (Figure 3, bottom left and right) was present in 6 of $8 \mathrm{MMC}$ (Figure 3, bottom left) and 6 of 9 OLO (Figure 3, bottom right) eyes - a specificity of $75 \%$ and $67 \%$, respectively.

The mean number of antiglaucoma medications was significantly reduced at the end point in both groups $(P=0.0001)$ : from $2.5( \pm 0.3)$ to $0.8( \pm 0.2)$ and from 2.6 $( \pm 0.2)$ to $0.9( \pm 0.2)$ in the MMC and OLO groups, respectively, without significant intergroup differences $(P=0.122)$.

Two patients in each group underwent the Carlo Traverso maneuver between the 1st and the 14th postoperative day. Laser suture lysis was performed between the first and the second postoperative week in four cases (20\%) in the MMC group and in three cases $(15 \%)$ in the OLO group, without intergroup difference. Bleb needling for encapsulated blebs was performed 
from one to four times in seven (35\%) and six (30\%) patients, respectively, again without intergroup difference.

The anterior chamber cells score was higher in OLO than in MMC group in the first postoperative day ( $2.4 \pm 0.8$ vs $1.8 \pm 0.6$, respectively, $P=0.018$ ), while the mean flare score was higher in MMC than in OLO group during the second postoperative week $(0.3 \pm 0.7$ vs 0 , respectively, $P=0.038$ ).

The frequency of postoperative complication did not significantly differ between the two groups (Table 5). Early bleb leakage was more frequent in the OLO than in the MMC group (3 vs 1 eye, respectively, $P=0.604$ ), while early hypotony was more frequent in MMC than in OLO group ( 8 vs 4 cases, respectively, $P=0.300$ ), with an increased frequency of choroidal detachment in the former (5 vs 2 cases, respectively, $P=0.407$ ). Two cases with not clinically significant avascular bleb (3a-1 according to MBGS) were noted in MMC group. No adverse reaction to the OLO, matrix extrusion, or conjunctival erosion was noted in OLO group.

\section{Discussion}

The need for alternative adjuvants to antimetabolites in glaucoma surgery arises from the difficult balance between loss of efficacy and postoperative complications related to hypotony. Early complications are mainly related to the procedure (eg, flaps fashioning, suturing technique, etc.), while late complications are caused by the prolonged fibroblast inhibition, with thin avascular blebs that are prone to leak. In our study, we applied one loose stitch in the OLO cases in order to counterbalance the pressure of the cylindrical implant over the scleral flap. This pressure could be indirectly confirmed by the first postoperative day's reduced tendency to hypotony in the OLO cases as compared with the MMC ones. OLO could therefore induce a modulation of the aqueous outflow occupying the subconjunctival space by its volume and applying a pressure on the top of the scleral flap.

There was no significant difference in the postoperative behavior between the two groups, with a highly significant and stable IOP reduction and very few antiglaucoma medications throughout the 24-month follow-up, indicating that the efficacy of the OLO

Table 5 Frequency (\%) of postoperative complications in the surgical groups

\begin{tabular}{lccl}
\hline & MMC group & OLO group & \multicolumn{1}{c}{$\mathrm{P}^{\mathrm{a}}$} \\
\hline Early hyphema & $1(5 \%)$ & $1(5 \%)$ & 1.0 \\
Early bleb leakage & $1(5 \%)$ & $3(15 \%)$ & 0.604 \\
Early hypotony & $8(40 \%)$ & $4(20 \%)$ & 0.300 \\
Choroidal detachment & $5(25 \%)$ & $2(10 \%)$ & 0.407 \\
\hline
\end{tabular}

Abbreviations: MMC, mitomycin-C; OLO, Ologen.

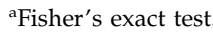

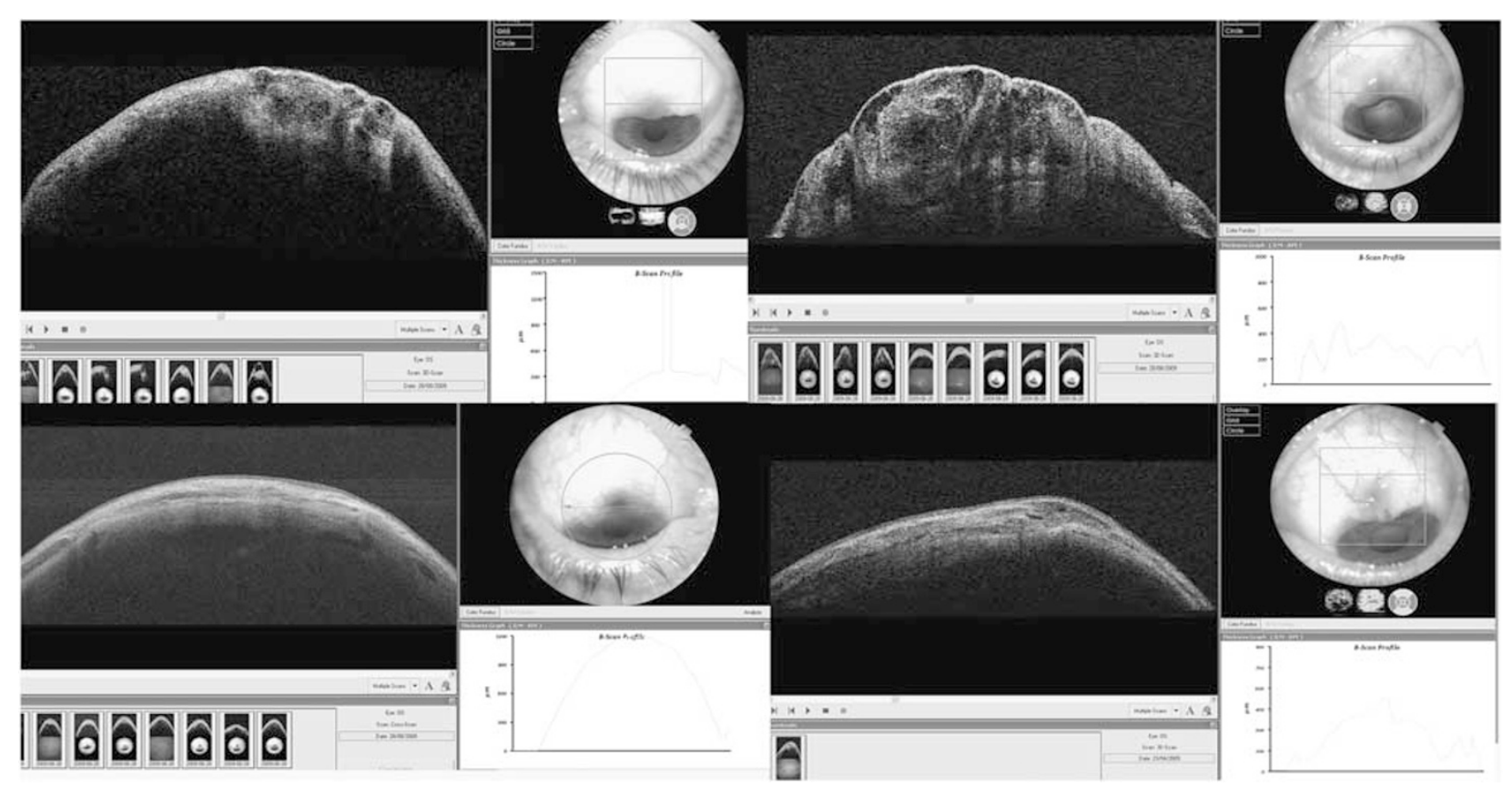

Figure 3 SD-OCT imaging of blebs postoperatively in eyes with or without complete success based on $\leq 17 \mathrm{~mm} \mathrm{Hg}$ target IOP. Successful ('thickened') blebs in eye with complete success (MMC adjuvant, top left; OLO adjuvant, top right). Failed ('non-thickened') blebs without complete success (MMC adjuvant, bottom left; OLO adjuvant, bottom right). 
implant is analogous to MMC. The similarity between OLO and MMC is further confirmed by their success rates at each target IOP level we considered.

The higher bleb height score in OLO group at the 3rd month could testify the persistence of the implant, whose volume could be added to the fluid-filled bleb spaces, and whose biodegradation, according to the manufacturer, can last a 6-month period. It is possible that a larger sample could have confirmed this difference in height for a longer period.

These data appear to be in contrast with the results of a recent paper where the bleb height at 60 days was lower and the vascularity bleb was higher in OLO group, where a lower success rate was noted when compared with MMC-treated eyes. ${ }^{31}$ This finding is discussed below.

In studies using either morphologic grading scale evaluation or experimental 3D anterior segment SD-OCT, bleb height is one of the parameters correlated with a lower IOP. ${ }^{28,32-34}$ While we observed a better bleb height persistence at one time in OLO group, we failed to demonstrate a better success rate. It is possible that the difference in bleb morphologic features was not large enough to significantly affect IOP control in the defined follow-up period.

The SD-OCT examination failed to show any qualitative difference in outer bleb appearance between the groups, indicating that the OLO implant neither enhances nor modifies the morphology of the outer layers of a functioning bleb. A larger sample size could confirm if the SD-OCT pattern of a successful bleb, besides confirming good test sensitivity, represents a prognostic factor for longer-term success.

The similar frequency of adjunctive postoperative procedures confirms the analogous performance of the intraoperative adjuvants.

The early, yet not significant, bleb leakage in OLO eyes can be ascribed to conjunctival flap limbal elevation induced by the implant volume, indicating a transitory mechanical effect.

The similar rates of postoperative complications in the two groups failed to reveal differences between the two adjuvants. The absence of cystic blebs and of the typical 'ring of steel' in both groups is partly due to the lack of significant reaction to the OLO insert with our therapeutic regimen and to the diffuse application and low dosage of MMC. The low complication rate with low-dosage MMC in our sample is in agreement with our previous studies. $8,24,35,36$

A recent pilot study ${ }^{20}$ is not in agreement with our results, since it showed OLO to have a larger amount of complications than simple trabeculectomy. The conjunctival closure with two simple 10-0 nylon sutures at the flap extremities was probably responsible for a higher incidence of positive Seidel test with flat anterior chamber in the study group, and could have reduced the beneficial effect of the implant in bleb development. Moreover, the reduced time of steroid administration, 1 month vs 2 months with variable regimen based on IPC criteria in our study, could have affected the vascularity control in OLO eyes, resulting in a hypothetical excess of fibroblast proliferation around and into the implant and higher end point IOP. The lower success rate and bleb functionality in OLO cases when compared with MMC ones, with a higher complication rate in the latter, reported in two studies, ${ }^{21,31}$ could hypothetically be ascribed to various factors such as small sample size, OLO size and manufacturer, concentration and modality of application of MMC, or postoperative therapeutic regimen.

Major limitations of our study consist, besides the small sample size, in the inclusion of PEXG, which is a 'secondary' glaucoma. On the other side, in our country the presence of PEXG is common ( $>30 \%$ ), and our sample adequately represents its prevalence in the general population.

In conclusion, our results suggest that the OLO implant could be a new, safe, and effective alternative to $\mathrm{MMC}$, with similar long-term success rate. The OLO implant may for instance be preferred when antimetabolite-related risks need to be avoided and may be useful in situations where IOP lowering and maximum safety are required, such as in high hyperopia, hemorrhagic risk, and monocularity. Due to our relatively small sample size, which limits the statistical comparison between the groups, further larger randomized trials are required to investigate the longterm efficacy and safety of this new device.

\section{Summary}

What was known before

- Trabeculectomy, introduced in 1968, remains the gold standard in the majority of eyes requiring glaucoma surgery.

- However, progressive loss of efficacy in some eyes remains an unsolved problem with this procedure.

- Many adjunctive modifications, such as antimetabolites or amniotic membrane transplantation have been tried in order to enhance and maintain the hypotensive effect of trabeculectomy.

\section{What this study adds}

- Our results suggest that the Ologen biodegradable collagen matrix implant could be useful as an adjuvant in trabeculectomy, and it is a new, safe, and effective alternative to MMC, with similar long-term success rate.

- The Ologen implant may for instance be preferred when antimetabolite-related risks need to be avoided and may be useful in situations where IOP lowering and maximum safety are required, such as in high hyperopia, hemorrhagic risk, and monocularity. 


\section{Conflict of interest}

The authors declare no conflict of interest.

\section{References}

1 Kitazawa Y, Kawase K, Matsushita H, Minobe M. Trabeculectomy with mitomycin: a comparative study with fluorouracil. Arch Ophthalmol 1991; 109: 1693-1698.

2 Skuta GL, Beeson CC, Higginbotham EJ, Lichter PR, Musch DC, Bergstrom TJ et al. Intraoperative mitomycin vs postoperative 5-fluorouracil in high-risk glaucoma filtering surgery. Ophthalmology 1992; 99: 438-444.

3 Smith MF, Doyle JW, Nguyen QH, Sherwood MB. Results of intraoperative 5-fluorouracil or lower dose mitomycin-C administration on initial trabeculectomy surgery. J Glaucoma 1997; 6: 104-110.

4 Fujishima H, Shimazaki J, Shinozaki N, Tsubota K. Trabeculectomy with the use of amniotic membrane for uncontrollable glaucoma. Ophthalmic Surg Lasers 1998; 29: 428-431.

5 Membrey WL, Poinoosawmy DP, Bunce C, Hitchings RA. Glaucoma surgery with or without adjunctive antiproliferatives in normal tension glaucoma: 1 intraocular pressure control and complications. Br J Ophthalmol 2000; 84: 586-590.

6 Singh K, Mehta K, Shaikh NM, Tsai JC, Moster MR, Budenz DL et al. Trabeculectomy with intraoperative mitomycin C vs 5-fluorouracil. Prospective randomized clinical trial. Ophthalmology 2000; 107: 2305-2309.

7 Yue J, Hu CQ, Lei XM, Qin GH, Zhang Y. Trabeculectomy with amniotic membrane transplantation and combining suture lysis of scleral flap in complicated glaucoma. Zhonghua Yan Ke Za Zhi 2003; 39: 476-480.

8 Cillino S, Zeppa L, Di Pace F, Casuccio A, Morreale D, Bocchetta F et al. E-PTFE (Gore-Tex) implant with or without low-dosage mitomycin-C as an adjuvant in penetrating glaucoma surgery: 2 year randomized clinical trial. Acta Ophthalmol 2008; 86: 314-321.

9 Palanca-Capistrano AM, Hall J, Cantor LB, Morgan L, Hoop J, WuDunn D. Long-term outcomes of intraoperative 5 -fluorouracil $v s$ intraoperative mitomycin $\mathrm{C}$ in primary trabeculectomy surgery. Ophthalmology 2009; 116: 185-190.

10 Wong TT, Khaw PT, Aung T, Foster PJ, Htoon HM, Oen FT et al. The Singapore 5-fluorouracil trabeculectomy study: effects on intraocular pressure control and disease progression at 3 years. Ophthalmology 2009; 116: 175-184

11 Palmer SS. Mitomycin as adjunct chemotherapy with trabeculectomy. Ophthalmology 1991; 98: 317-321.

12 Jampel HD, Friedman DS, Lubomski LH, Kempen JH, Quigley $\mathrm{H}$, Congdon $\mathrm{N}$ et al. Effect of technique on intraocular pressure after combined cataract and glaucoma surgery. An evidence-based review. Ophthalmology 2002; 109: 2215-2224.

13 Wilkins M, Indar A, Wormald R. Intra-operative mitomycin C for glaucoma surgery. Cochrane Database Syst Rev 2005; 4 CD002897.

14 Anand N, Atherley C. Deep sclerectomy augmented with mitomycin C. Eye 2005; 19: 442-450.

15 Anand N, Arora S, Clowes M. Mitomycin C augmented glaucoma surgery: evolution of filtering bleb avascularity, transconjunctival oozing, and leaks. Br J Ophthalmol 2006; 90: $175-180$.

16 Hsu WC, Spilker MH, Yannas IV, Rubin PA. Inhibition of conjunctival scarring and contraction by a porous collagen-glycosaminoglycan implant. Invest Ophthalmol Vis Sci 2000; 41: 2404-2411.

17 Chen HS, Ritch R, Krupin T, Hsu WC. Control of filtering bleb structure through tissue bioengineering: an animal model. Invest Ophthalmol Vis Sci 2006; 47: 5310-5314.

18 Hsu WC, Ritch R, Krupin T, Chen HS. Tissue bioengineering for surgical bleb defects: an animal study. Graefes Arch Clin Exp Ophthalmol 2008; 246: 709-717.

19 Aptel F, Dumas S, Denis P. Ultrasound biomicroscopy and optical coherence tomography imaging of filtering blebs after deep sclerectomy with new collagen implant. Eur J Ophthalmol 2009; 19: 223-230.

20 Papaconstantinou D, Georgalas I, Karmiris E, Diagourtas A, Koutsandrea C, Ladas I et al. Trabeculectomy with OloGen vs trabeculectomy for the treatment of glaucoma: a pilot study. Acta Ophthalmol 2010; 88: 80-85.

21 Rosentreter A, Schild AM, Jordan JF, Krieglstein GK, Dietlein TS. A prospective randomised trial of trabeculectomy using mitomycin $\mathrm{C} v \mathrm{~s}$ an ologen implant in open angle glaucoma. Eye (Lond) 2010; 24: 1449-1457.

22 Stalmans I, Gillis A, Lafaut AS, Zeyen T. Safe trabeculectomy technique: long-term outcome. $\mathrm{Br} J$ Ophthalmol 2006; 90: 44-47.

23 Dhingra S, Khaw PT. The Moorfields safer surgery system. Middle East Afr J Ophthalmol 2009; 16: 112-115.

24 Cillino S, Di Pace F, Casuccio A, Lodato G. Deep sclerectomy vs punch trabeculectomy: effect of low-dosage mitomycin C. Ophthalmologica 2005; 219: 281-286.

25 Marquardt D, Lieb WE, Grehn F. Intensified postoperative care us conventional follow-up: a retrospective long-term analysis of 177 trabeculectomies. Graefes Arch Clin Exp Ophthalmol 2004; 242: 106-113.

26 Traverso CE, Greenidge KC, Spaeth GL, Wilson RP. Focal pressure: a new method to encourage filtration after trabeculectomy. Ophthalmic Surg 1984; 15: 62-65.

27 Wells AP, Ashraff NN, Hall RC, Purdie G. Comparison of two clinical Bleb grading systems. Ophthalmology 2006; 113: 77-83.

28 Kawana K, Kiuchi T, Yasuno Y, Oshika T. Evaluation of trabeculectomy blebs using 3-dimensional cornea and anterior segment optical coherence tomography. Ophthalmology 2009; 116: 848-855.

29 Singh M, See JL, Aquino MC, Thean LS, Chew PT. High-definition imaging of trabeculectomy blebs using spectral domain optical coherence tomography adapted for the anterior segment. Clin Exp Ophthalmol 2009; 37: 345-351.

30 Brennan P, Silman A. Statistical methods for assessing observer variability in clinical measures. BMJ 1992; 304: 1491-1494.

31 Boey PY, Narayanaswamy A, Zheng C, Perera SA, Htoon HM, Tun TA et al. Imaging of blebs after phacotrabeculectomy with Ologen collagen matrix implants. Br J Ophthalmol 2011; 95: 340-344.

32 Picht G, Grehn F. Classification of filtering blebs in trabeculectomy: biomicroscopy and functionality. Curr Opin Ophthalmol 1998; 9: 2-8.

33 Cantor LB, Mantravadi A, WuDunn D, Swamynathan K, Cortes A. Morphologic classification of filtering blebs after glaucoma filtration surgery: the Indiana Bleb Appearance Grading Scale. J Glaucoma 2003; 12: 266-271. 
34 Lopes JF, Moster MR, Wilson RP, Altangerel U, Alvim HS, Tong MG et al. Subconjunctival sodium hyaluronate $2.3 \%$ in trabeculectomy: a prospective randomized clinical trial. Ophthalmology 2006; 113: 756-760.

35 Cillino S, Di Pace F, Casuccio A, Calvaruso L, Morreale D, Vadalà $\mathrm{M}$ et al. Deep sclerectomy vs punch trabeculectomy with or without phacoemulsification: a randomized clinical trial. J Glaucoma 2004; 13: 500-506.

36 Cillino S, Di Pace F, Casuccio A, Cillino G, Lodato G. Deep sclerectomy vs trabeculectomy with low-dosage mitomycin C: four-year follow-up. Ophthalmologica 2008; 222: $81-87$. 